\title{
Design and Evaluation of Chitosan films for Transdermal delivery of Glimepiride
}

\author{
Available online at www.ijistweb.com \\ RESEARCH ARTICLE \\ Syed Ata Ur Rahman*1, Neeraj Sharma ${ }^{2}$ \\ ${ }^{1}$ College of Pharmacy, Sri Satya Sai University of Technology \& Medical Sciences, Pachama, Sehore
}

(M.P.) 466001, India

${ }^{2}$ School of Pharmacy, Madhyanchal Professional University, Ratibad, Bhopal, 462044, India

*Corresponding Author's E-mail: om11agra85@gmail.com

\begin{abstract}
Glimepiride is a third generation oral antidiabetic sulphonylurea drug frequently prescribed to patients of type 2 diabetes. However, its oral therapy is encountered with bioavailability problems due to its poor solubility leading to irreproducible clinical response, in addition to adverse effects like dizziness and gastric disturbances. As a potential for convenient, safe and effective antidiabetic therapy, the rationale of this study was to develop a transdermal delivery system for glimepiride. Chitosan polymer was utilized in developing transdermal films for glimepiride. Chitosan has film forming ability, bioadhesive and absorption enhancing properties. Aiming at optimizing the drug delivery and circumventing the skin barrier function, inclusion complexation of glimepiride with beta-cyclodextrin ( $\beta$ $\mathrm{CyD}$ ) as well as the use of several conventional penetration enhancers were monitored for augmenting the drug flux. The physical and mechanical properties of the prepared films were investigated using tensile testing, IR spectroscopy and X-ray diffractometry. Release studies revealed adequate release rates from chitosan films. Permeation studies through full thickness rat abdominal skin were conducted. High flux values were obtained from films comprising a combination of the drug with limonene and ethanol as well as from films containing glimepiride- $\beta$-CyD complex. In vivo studies on diabetic rats for selected formulae revealed a marked therapeutic efficacy sustained for about 48 hours. The above-mentioned results shed light on feasibility of utilizing chitosan as an effective, safe transdermal delivery system for glimepiride characterized by increased patient compliance and better control of the disease.
\end{abstract}

Keywords: Glimepiride, transdermal, chitosan, penetration enhancers, cyclodextrins.

\section{Introduction}

Glimepiride is a new third generation sulphonylurea oral hypoglycaemic agent. It has been proposed for the treatment of type-2 diabetes, whenever blood glucose levels cannot be adequately controlled by diet, physical exercise and weight reduction alone. Glimepiride enhances the normal action of insulin on peripheral glucose uptake (insulinsensitizing effect). It also enhances peripheral glucose uptake and inhibits glucose output (insulin-mimetic effects). It directly increases the number of glucose transporters in the plasma membranes of muscle and fat cell. In comparison to other sulphonylurea antidiabetic drugs, glimepiride expresses an insulin-sparing effect and minimal interaction with the cardiovascular system $(1,2)$.

However, current glimepiride formulations have some adverse effects and bioavailability problems. Its bioavailability problems are encountered because of its poor $\mathrm{pH}$ dependent solubility. This poor aqueous solubility
$(0.001908 \mathrm{mg} / \mathrm{ml})$ and hence slow dissolution may lead to irreproducible clinical response; high inter individual variations and inappropriate maintenance of blood levels of the drug and glucose. In some cases, therapeutic failure occurs due to subtherapeutic plasma drug levels (3-5). The most frequently reported adverse events are hypoglycemia, dizziness and headache. Moreover, oral therapy may cause gastric disturbances, heartburn, nausea and diarrhea which greatly affect patient compliance (6).

Poor compliance with medication is a major barrier to successful patient management in type 2 diabetes. Establishing and maintaining effective glycemic control in patients reduce the risk of long-term microvascular and macrovascular complications.

New anti-diabetic regimens are urgently required. One possibility involves drug delivery across the skin. Transdermal delivery could be 
an alternative route to overcome many of the problems associated with the oral therapy. Recently, the transdermal route has vied with oral treatment as the most successful innovative research area in drug delivery (7).

Advantages of the transdermal delivery over other forms of administration include improved patient compliance due to less frequent dosing, avoidance of the hostile gastrointestinal environment and its impact not only on the drug absorption but also on its stability, avoidance of first pass effect, maintenance of uniform systemic drug within the therapeutic level for prolonged time, reduction of inter- and intrapatient variability, termination of the drug input simply by the removal of the patch (8).

Recently, different oral antidiabetic sulphonylurea drugs have been subjected to extensive investigations for their appropriateness to be delivered via transdermal route e.g., glibenclamide, chloropropamide (9-11), gliquidone (12) and glipizide $(13,14)$. The results were very promising. However, the literature revealed that the studies on transdermal delivery of glimepiride were very rare. Glimepiride was the subject of a single study aimed at development of a transdermal system (15). The study investigated the pharmacokinetics and relative bioavailability of glimepiride gelmatrix patch in rats; the results revealed that the patch exhibited good controlled-release and bioavailability properties.

The importance of glimepiride, in spite of its oral administration problems in the aspect of its unique benefits compared to other members of the oral antidiabetic sulphonylurea group, motivated our study to design an acceptable, simple, safe, economic and prolonged release transdermal formula for glimepiride.

In the current research, the use of chitosan was inspected for potentiality to develop a transdermal delivery system for glimepiride. Chitosan molecule is a copolymer of $\mathrm{N}$-acetylD-glucosamine and D-glucosamine, which are constituents of normal mammalian tissue, thus provides biocompatibility. In recent years chitosan has gained increasing interest in pharmaceutical field due to its favorable biological properties such as biodegradability and lack of toxicity, together with its wide availability in nature and low cost $(16,17)$. From the biopharmaceutical view point, chitosan has an original characteristic of mucoadhesion ${ }^{18}$. It has film forming and gelation abilities, along with its absorption enhancing properties and versatility in development of several drug delivery systems (19). It enhances the paracellular drug transport via transient opening of tight junction between epithelial cells (20, 21). Furthermore, it has demonstrated its effectiveness in improving dissolution properties and bioavailability of poorly-soluble drugs (22).

Vehicles designed to enhance drug delivery through the skin must incorporate specific elements that improve the ability of the delivery system to overcome the barrier posed by the stratum corneum (23). Thus, we investigated inclusion complexation of glimepiride with $\beta$ cylodextrin in view of its ability to improve the solubility of the drug. It was stated that the solubility of glimepiride was increased markedly by complexation with $\beta-\mathrm{CyD}$. The solubilizing power, calculated as mole glimepiride solubilized per one mole of cyclodextrin, was 0.003 (24). The solubility study had revealed the existence of a phase solubilizing diagram of the Ap type indicating the formation of high order complex between the drug and the cyclodextrin (24). Cyclodextrins are able to form both inclusion and non-inclusion complexes. These complexes are also able to solubilize lipophilic water insoluble drugs through inclusion complexation in addition to non-inclusion complexation or micelle-like structure formation.

Also, CyDs are able to increase dermal and transdermal drug delivery without affecting the barrier function of the skin (25-28). In addition, various conventional penetration enhancers belonging to different chemical groups were monitored to assess their penetration enhancing effect towards the drug. The developed formulae of the drug were evaluated for permeation, as well as for their impact on the biological performance of the drug.

\section{Materials and Methods}

\subsection{Materials}

Glimepiride was kindly provided by Delta Pharma. Co. (Mumbai, India). Chitosan (MW 150,000 , deacetylation degree 75-85\%), $\beta$ cyclodextrin, cineole, menthol, limonene and streptozotocin were purchased from Sigma 
Chemical Company (St. Louis, USA). Oleic acid was purchased from Fluka (Germany). Sodium dihydrogen phosphate was supplied from S. D. fine-chem. Ltd. (Mumbai, India) and disodium hydrogen phosphate was purchased from BDH Laboratory Supplies (Poole England). Lactic acid, acetic acid and ethanol were supplied from Adwic, Banglore. All other ingredients are of analytical grade.

\subsection{Methods}

\subsubsection{Elucidation of the Stoichiometric Ratio and Preparation of Glimepiride- $\beta$ - Cyclodextrin Complex}

The continuous variation method (29) was utilized to determine the stoichiometric ratio of glimepiride- $\beta-\mathrm{CyD}$ complex by spectrophotometric measurements. The absorbance values of fixed total concentration $(0.072 \mathrm{mmol} / \mathrm{l})$ of glimepiride and $\beta$-CyD were measured at $226 \mathrm{~nm}$. If it was found that the absorbance values of these solutions were different from the sum of the corresponding values of their components. Then, the observed absorbance difference was plotted against mole fraction.

Inclusion complex of glimepiride in $\beta-\mathrm{CyD}$ was prepared by the kneading method (30), whereby glimepiride was added to the cyclodextrin in a molar ratio equivalent to its corresponding stoichiometric ratio in the complex, kneaded thoroughly with least amount of water to obtain a paste which was then dried under vacuum at room temperature in presence of phosphorus pentoxide as a drying agent.

\subsubsection{Preparation of Chitosan Films}

Chitosan $(1.5 \% \mathrm{w} / \mathrm{v})$ was dissolved in water containing $2 \% \mathrm{w} / \mathrm{v}$ of a $1: 1$ mixture of lactic acid and acetic acid solution and stirred overnight using a magnetic stirrer. The resulting solution was filtered through a sintered glass filter to remove the extraneous matter. The resulting solution was medicated with the drug or its equivalent amount of the glimepiride complex followed by sonication for 2 hours. Two concentrations of glimepiride were used in preparing the films. The first was $0.30 \% \mathrm{w} / \mathrm{w}$ and the second was $0.125 \% \mathrm{w} / \mathrm{w}$. The first concentration, suitable for human, was used for preparing films having a dose of $\left(6 \mathrm{mg} / 3 \mathrm{~cm}^{2}\right)$ which were used for in vitro evaluation. The second concentration was used in preparing films comprising a dose $\left(2.5 \mathrm{mg} / 3 \mathrm{~cm}^{2}\right)$, suitable for rats, these films were used for in-vivo assessment. In case of investigating penetration enhancers, they were added at this step in the corresponding concentrations followed by sonication for 1 hour. The mixtures were then casted on teflon plates and dried in an incubator at $25^{\circ} \mathrm{C}$ for 24 hours. The spreading volume was $0.9 \mathrm{ml} / \mathrm{cm}^{-2}$. The mixture was spread uniformly. After drying, the films were carefully peeled off. The films, with uniform thickness $=200 \pm 25$ $\mu \mathrm{m}$, were stored in tight glass containers maintained at room temperature until further investigations.

\subsubsection{Measurement of Mechanical Properties of Chitosan Films}

The mechanical properties (tensile strength and elongation at break) were measured at room temperature on an electric tensile testing machine (Zwick 1425) according to ASTM D 412 (31). The tensile strength and elongation at break were calculated as follows:

Tensile strength $\left(\mathrm{N} / \mathrm{mm}^{2}\right)=$ Breaking force $(\mathrm{N})$ $/$ Cross sectional area $(\mathrm{mm})^{2}$

Elongation at break \% $=($ Increase in length at break (mm) /Original length) x100)

\subsubsection{Characterization of Glimepiride - Chitosan Films}

\subsubsection{IR Studies}

The IR spectra were recorded using a Shimadzu 435 U-O4 IR spectrophotometer by $\mathrm{KBr}$ disc method. Prior to measurement, the films were cut and then ground with a small vibrating mill (Shimadzu vibrating mill, Japan) before grinding with $\mathrm{KBr}$.

\subsubsection{X-Ray Diffraction Studies}

The X-ray diffractograms were recorded at room temperature using a Diano X-ray diffractometer equipped with Co K $\alpha$. The tube operated at $45 \mathrm{kV}, 9 \mathrm{~mA}$. The dried film of uniform thickness was carefully placed on glass slide and scanned in the $2 \theta$ range of $5-80^{\circ}$.

\subsubsection{Release Studies}

Chitosan films, of $3.0 \mathrm{~cm}^{2}$ surface area, comprising $6 \mathrm{mg}$ of glimepiride or its equivalent amount of glimepiride- $\beta$-CyD complex were applied on a glass slide and covered with 
stainless steel mesh screen and clamped together (14). The assembly was placed at the bottom of the USP dissolution tester (Erweka Apparatebau $\mathrm{GmbH}$, model DT-D, Germany). The release studies were carried out according to the paddle method (32). The height of the paddle from the surface of the assembly was adjusted to $2.5 \mathrm{~cm}$. The vessel contained $900 \mathrm{ml}$ phosphate buffer solution $(\mathrm{pH}=5.5)$, the temperature was adjusted at $32^{\circ} \mathrm{C}$ and the speed at $50 \mathrm{rpm}$. Aliquots of 5 $\mathrm{ml}$ were withdrawn from the release medium at each time interval through sintered glass filter and replaced by equivalent amounts of the buffer solution. The amount of drug released from the bases was determined spectrophotometrically at $226 \mathrm{~nm}$ using Shimadzu UV Spectrophotometer (2401/PC), Japan. Blank experiments were simultaneously performed. The amount of drug released per unit surface area was plotted against time and the release rate was calculated and statistically analyzed using the T-test for statistical analysis. This statistical analysis was computed using the SPSS ${ }^{\circ}$ software.

\subsubsection{Permeation Studies}

Permeation studies were carried for chitosan films comprising glimepiride alone or as a $\beta$ CyD complex. In addition, different penetration enhancers were assessed for their impact on drug permeation. These enhancers comprised: oleic acid, cineol, limonene, menthol and ethanol.

Male Wistar rats (200 to $250 \mathrm{~g}$ ) were sacrificed, the abdominal skin was excised and the adhering fat eliminated. The abdominal hair was removed carefully using electric razors. The whole thickness skin was equilibrated in phosphate buffer solution $(\mathrm{pH}=7.4)$ for $1 \mathrm{hr}$ before the beginning of each experiment. The skin used was of thickness $0.8 \pm 0.05 \mathrm{~mm}$. The prepared films of $3.0 \mathrm{~cm}^{2}$ surface area, comprising $6 \mathrm{mg}$ of glimepiride or its equivalent amount of glimepiride- $\beta$-CyD complex, with or without conventional penetration enhancers, were applied firmly on the epidermis to assure adhesiveness. Skin was mounted on vertical Franz-type diffusion cell with the dermis facing the receptor compartment. The receptor compartment was filled with phosphate buffer solution $(\mathrm{pH}=7.4)$. Then, the donor and receptor compartments were clamped together. The donor compartment was covered with parafilm. The jacketed cells were circulated with thermostated water maintained at $37^{\circ} \mathrm{C}$. The temperature was maintained at $37 \pm 0.5{ }^{\circ} \mathrm{C}$ and the receptor compartment, $50 \mathrm{ml}$ volume capacity, was constantly stirred at $300 \mathrm{rpm}$. Samples of receptor fluid $(1 \mathrm{ml})$ were withdrawn periodically, up to 24 hours, and replenished with fresh buffer solution. The samples were assayed for the drug spectrophotometrically at $226 \mathrm{~nm}$. Blank experiments for the investigated films free from drug were carried concurrently. The amount of drug was measured against the blank. Steady-state flux was estimated from the slope of the straight-line portion of the cumulative amount of drug permeated per $\mathrm{cm} 2$ of the patch against time profile. The data were analyzed statistically by the one-way ANOVA test followed by the least significant difference procedure. This statistical analysis was computed using the SPSS ${ }^{\circledR}$ software.

\subsubsection{Assessment of Therapeutic Efficacy}

Therapeutic efficacy assessment was conducted on streptozotocin- induced diabetic rats, whereby the decrease in blood glucose level (BGL) was taken as a pharmacodynamic parameter for evaluating the bioavailability of the drug (33). Male Wistar rats weighing 200$250 \mathrm{~g}$ were kept on standard diet and maintained under controlled conditions of humidity (30$70 \%)$ and temperature $\left(24 \pm 2^{\circ} \mathrm{C}\right)$. The animals were fasted overnight and then intraperitoneally injected with $50 \mathrm{mg} / \mathrm{kg}$ streptozotocin $(34,35)$ 10-14 days prior to the study for inducing diabetes. Fasting blood glucose level was assessed using Fast Take glucometer SmartScan ${ }^{\circledR}$ (36). Rats having moderate diabetes, with fasting BGL in the range of 200$300 \mathrm{mg} / \mathrm{dl}$, were selected for the study. The animals were divided into 5 groups each comprising 12 rats. The first group was given the drug orally in a dose of $10 \mathrm{mg} / \mathrm{kg}$ body weight (37). Chitosan film of surface area $3.0 \mathrm{~cm}^{2}$ comprising a dose of $2.5 \mathrm{mg}$ glimepiride (formula 1) was applied tightly to ensure its adhesiveness on the shaved back region of the animals of the second group. Then, it was covered with a piece of aluminum foil and an adhesive tape. The same formula in presence of a combination of $5 \%$ limonene together with $10 \%$ ethanol (formula 2) was applied to the third group. The fourth group was subjected to transdermal application of the chitosan film comprising glimepiride- $\beta-\mathrm{CyD}$ complex 
equivalent to $2.5 \mathrm{mg}$ glimepiride (formula 3 ). In addition, a plain chitosan film of the same size was applied on the animals of the fifth group. The blood glucose level (BGL) after oral drug administration and after transdermal application of the investigated films was measured at different time intervals, up to $48 \mathrm{~h}$. Each animal served as its own control and hence, the hypoglycemic response was evaluated as percentage decrease in blood glucose level calculated as follows:

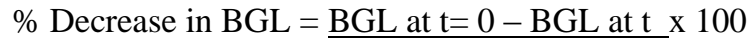 BGL at $\mathrm{t}=0$}

The pharmacodynamic parameters taken into consideration were maximum percentage decrease in blood glucose level, time for maximum response $\left(t_{\max }\right)$, time at which half peak percentage decrease in BGL prevails $\left(t_{1 / 2} \mathrm{p}\right)$ and area under percentage decrease in BGL versus time curve (AUC0-48 h) which was calculated adopting the trapezoidal rule (38).

Statistical analysis of the results was performed using one-way analysis of variance (ANOVA), followed by the least-significant difference test (LSD). This statistical analysis was computed using the SPSS ${ }^{\circledR}$ software.

All Animal studies were conducted according to the protocol approved by the Institutional Animal Ethics Committee, College of Pharmacy, Sri Satya Sai University of Technology \& Medical University, Sehore, Madhya Pradesh, India.

\section{Results and Discussion}

\subsection{Elucidation of the Stoichiometric Ratio and Preparation of Glimepiride- $\beta$ - Cyclodextrin Complex}

The absorbance values of fixed total concentration $(0.072 \mathrm{mmol} / \mathrm{l})$ of glimepiride and $\beta$-CyD were measured at $226 \mathrm{~nm}$. It was found that the absorbance values of these solutions were not the same as the sum of the corresponding values of their components. The calculated absorbance difference was plotted against mole fraction. For a constant total concentration of the two species of a complex, the complex is in its highest concentration at the point where the two species are combined in the ratio in which they occur in the complex. Abrupt change in absorbance difference existed at 0.33 drug mole fraction indicating formation of high order complex having stoichiometric ratio of 1 drug: 2 cyclodextrin.

In general, maximal absorption enhancement is obtained when just enough cyclodextrin is used to solubilize the drug. Only the free form of the drug, which is in equilibrium with the complexed one, is capable of penetrating lipophilic barriers and eventually entering the systemic circulation. Further addition of cyclodextrin decreases the free fraction of the drug and, hence, reduces the drug's bioavailability. Thus, glimepiride was added to $\beta-C y D$ in this molar ratio to prepare an inclusion complex by the kneading method $(24,30)$.

\subsection{Measurement of Mechanical Properties of Chitosan Films}

The tensile testing provides an indication of the strength and elasticity of the film, which can be reflected by tensile strength and elongation at break.

The prepared chitosan films showed a tensile strength at break equal to $30.88 \pm 2.41 \mathrm{~N} / \mathrm{mm}^{2}$ (mean \pm S.E., $\mathrm{n}=3$ ) and elongation $\%$ equal to $78.32 \pm 1.56$. This depicts that the prepared chitosan films possess reasonable tensile properties.

The strength property was provided from the use of acetic acid as a solvent for chitosan and the elasticity was gained by the use of lactic acid which is commonly used as solvent and at the same time as a plasticizer.

Lactic acid could be used as a universal solvent for chitosan (39) and has been employed as a plasticizer to improve the flexibility of films (40). The effects of plasticization are the result of the plasticizer's ability to weaken polymeric intermolecular attractions, thus allowing the polymer molecules to move more readily which increases the flexibility of the polymer (41).

The elasticity of the prepared chitosan films would improve the contact between the film and the tissue, hence promoting penetration of the polymeric chains into the skin tissue. This leads to increase in the adhesion strength (42). For chitosan film to be applied topically, it should be durable, stress resistant, flexible, pliable, and elastic. It should be easy to apply and remove without incurring any upset. Moreover, the film must be rapidly and uniformly adherent to conform appropriate contact to the skin (43). 


\subsection{Characterization of Glimepiride - Chitosan Films}

\subsubsection{Infrared Spectroscopy}

Fig. (1) depicts I.R. spectra of glimepiride and the investigated chitosan films. The IR spectra reveal that it has major peaks at $3369 \mathrm{~cm}^{-}$
1 due to amide stretching and at $1673 \mathrm{~cm}^{-1}$ corresponding to $\mathrm{N}-\mathrm{H}$ bending. The carbonyl group stretching peak appears at $1708 \mathrm{~cm}^{-1}$. In addition, several peaks of $-\mathrm{C}-\mathrm{H}$ and $=\mathrm{C}-\mathrm{H}$ bending in the frequency range 600 to $1500 \mathrm{~cm}^{-1}$ appears in the spectrum.

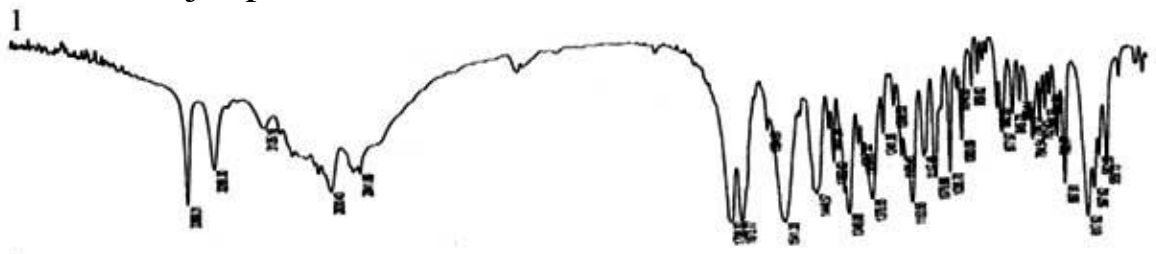

2
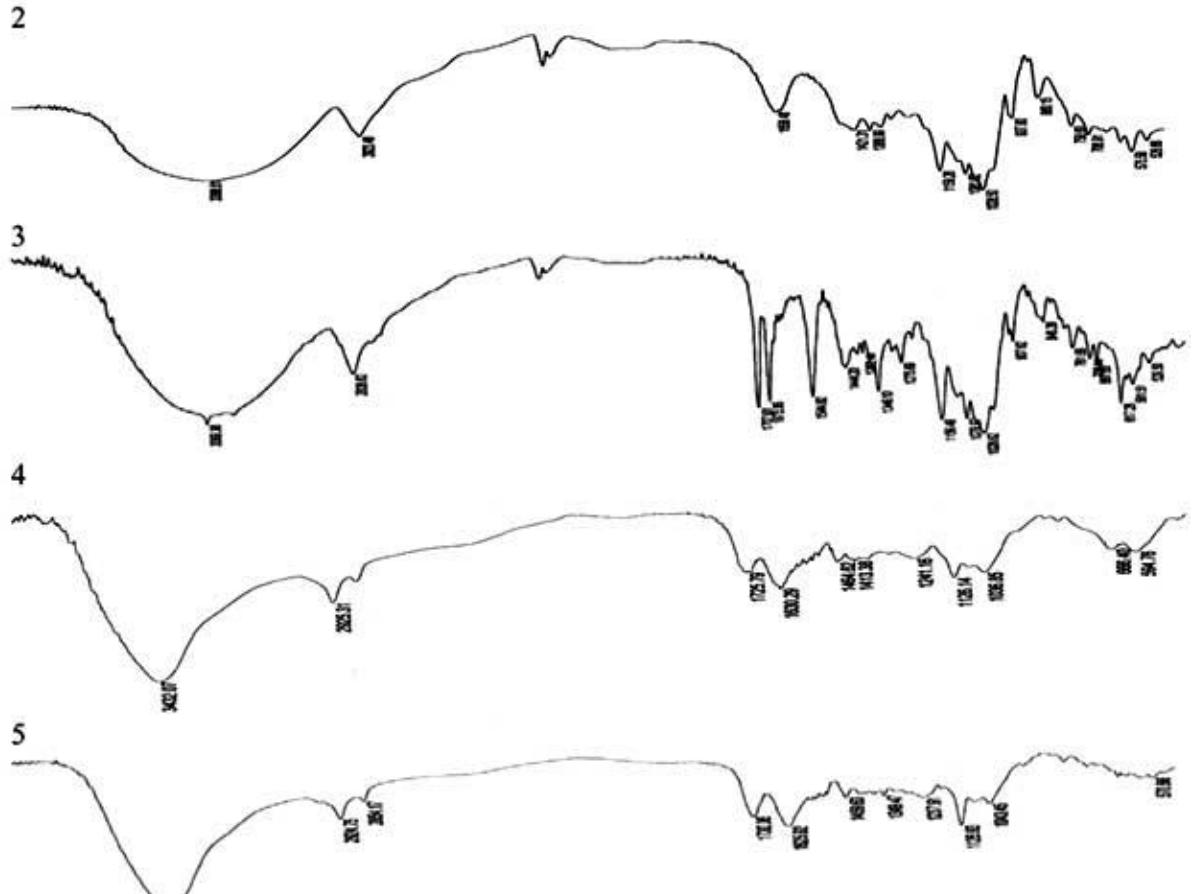

6

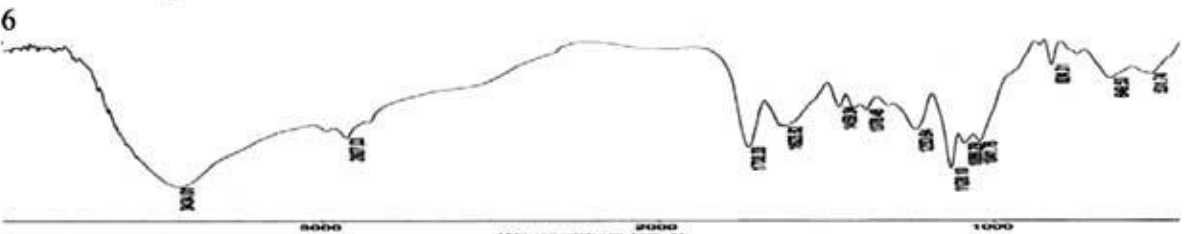

Figure 1. IR spectra of glimepiride and the investigated chitosan films 1: Glimepiride; 2 : $\beta$-CyD; 3 : glimepiride- $\beta-\mathrm{CyD} ; 4$ : chitosan film comprising glimepiride- $\beta-\mathrm{CyD} ; 5$ : chitosan film comprising glimepiride; 6: chitosan film

The IR spectrum of the investigated $\beta-\mathrm{CyD}$ is characterized by an intense broad band at $3388 \mathrm{~cm}^{-1}$ due to $\mathrm{O}-\mathrm{H}$ stretching vibrations and $\mathrm{O}-\mathrm{H}$ bending appears at $1658 \mathrm{~cm}^{-1}$. The stretching vibrations of the $=\mathrm{C}-\mathrm{H}$ and $\mathrm{C}-\mathrm{H}$ groups appear in the $2800-3000 \mathrm{~cm}^{-1}$ region.

The IR spectrum of glimepiride- $\beta-C y D$ complex shows considerable differences when compared with those of their corresponding constituents. A decrease in frequency of a specific peak is generally seen on complexation (44), suggesting that after the formation of the inclusion complex, existing bonds were broken and also reduced in their intensities (45). Similarly the N-H stretching band of the amide exhibited broadening when the inclusion complex was formed. These modifications clearly indicate the presence of host-guest interactions and suggest the formation of hydrogen bonds between glimepiride and the CyD $(46,47)$. 
The IR spectrum of chitosan film exhibited broad peak at $3434 \mathrm{~cm}^{-1}$ assigned to $\mathrm{OH}$ stretching, indicating intermolecular hydrogen bonding of chitosan molecules. The N-H stretching band appears at $2927 \mathrm{~cm}^{-1}$, also carbonyl stretching appears at $1733 \mathrm{~cm}^{-1}$. In addition, $\mathrm{N}-\mathrm{H}$ and $\mathrm{O}-\mathrm{H}$ bending bands appear at $1623 \mathrm{~cm}^{-1}$.

In case of the chitosan films comprising glimepiride or glimepiride-CyD complex, the sharp peaks corresponding to the amide group stretching of glimepiride are not detectable. In addition, the hydroxyl and amine bending bands of the pure chitosan film are shifted from their original positions with disappearance of some peaks and formation of new ones. This indicates sort of interaction between the drug and the polymer.

\subsubsection{X-Ray Diffractometry}

1

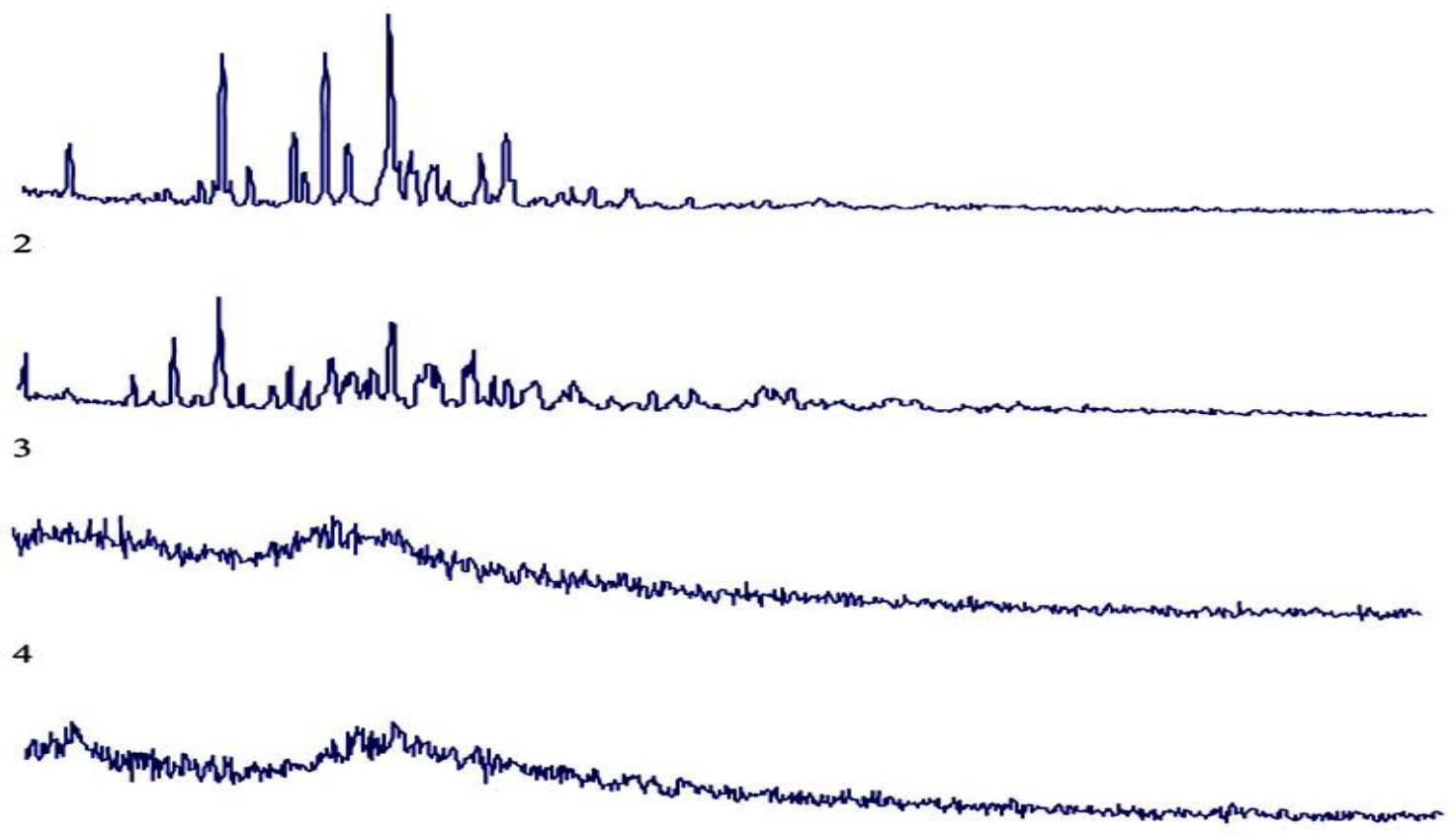

5

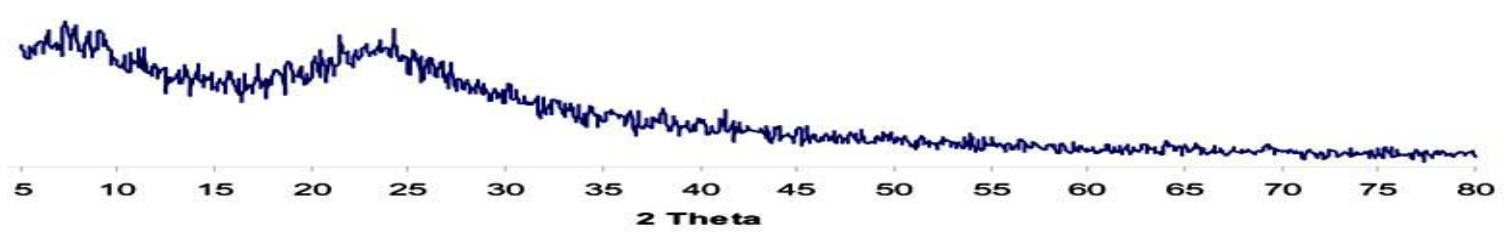

Figure 2. X-ray diffraction patterns of glimepiride and the investigated chitosan films

1: Glimepiride; 2: glimepiride- $\beta-\mathrm{CyD} ; 3$ : chitosan film comprising glimepiride- $\beta$ - CyD; 4 : chitosan film comprising glimepiride; 5: chitosan film
The X-ray diffraction pattern of glimepiride (Fig. 2) showed sharp diffraction patterns reflecting its crystallinity; the highest peak was detectable at the 2 theta value of $24.5^{\circ}$.

The diffractogram of the drug-CyD complex (Fig. 2) apparently differed from that of the drug and gave somewhat diffuse diffraction pattern suggesting that it is less crystalline than the drug per se. This was further evidenced from the data of the degree of crystallinity of the complex which was less than that of the drug. Crystallinity can be determined by comparing some representative peak heights in the diffraction patterns of the complex with those of a reference. The relationship used for the calculation of crystallinity was relative degree of crystallinity (RDC). RDC $=I_{\text {sam }} / I_{\text {ref }}$, where $I_{\text {sam }}$ is the peak height of the sample under investigation and $I_{\text {ref }}$ is the peak height at the same angle for the reference with the highest intensity (48). 
For the prepared chitosan film comprising glimepiride or its CyD complex, their diffractograms contained no sharp peaks associated with the crystalline glimepiride molecule. These diffraction patterns were nearly similar to those of the pure chitosan film, suggesting that the drug was either dissolved in the polymer or present in an amorphous state within the polymeric matrix. Polymers contain randomly entangled chains; such an irregular molecular structure prevents crystallization (49).

\subsection{Release Studies of Glimepiride from Chitosan Films}

Release studies of the films are very crucial, because one needs to maintain the drug concentration on the surface of stratum corneum consistently and substantially greater than the drug concentration in the body, to achieve a constant rate of drug permeation.

The study revealed that the $\%$ drug released from the chitosan film comprising glimepiride and that comprising glimepiride- $\beta$ - $\mathrm{CyD}$ were adequate, reaching $39.68 \pm 1.73$ and $61.50 \pm$ 2.02 within 6 hours, respectively (mean \pm S.E., $n$ $=3$ ). The release - rate values revealed that complexation of the drug within $\beta$-CyD leads to significant enhancement $(\mathrm{p}<0.005)$ of the release rate from $0.185 \pm 0.007$ (mg.cm-2.h-1) to $0.242 \pm$ $0.008\left(\mathrm{mg} . \mathrm{cm}-2 \cdot \mathrm{h}^{-1}\right)$.

The results of the investigation suggest that the enhancing effect of cyclodextrin on drug release from the film can be explained by the increase in solubility of the drug by $\mathrm{CyD}$ complexation (24), thus improving the diffusible form of the drug species at the skin base interface. As the concentration gradient over the diffusion layer is the main driving force for the drug delivery from the base to the surface of the barrier, the availability of dissolved drug is crucial for effective drug delivery (50). This is also consistent with those reports pointing out that the release of drugs from vehicles was improved by complexation with cyclodextrins (14, 51-53).

\subsection{Permeation Studies}

To achieve and maintain therapeutic concentration of drug in blood, the resistance of the skin to diffusion of drugs has to be reduced so that drug molecules can cross skin and maintain therapeutic levels in blood. One longstanding approach for improving transdermal drug delivery uses penetration enhancers which penetrate into skin to reversibly decrease the barrier resistance (54).

The permeation profiles of glimepiride from chitosan films comprising the drug alone and in presence of different penetration enhancers are presented in Table $\mathbf{1}$ and Fig. (3). Enhancement of the permeation rate of the drug by complexation of the drug with $\beta$ - $\mathrm{CyD}$ was evident. This enhancement is significant $(p<0.001)$. The effect of the CyD on the permeation rate of drugs through the skin may also be attributed to the thermodynamic activity of the drug in the vehicle $(50,55)$. It is generally recognized that CyDs act as true carriers by keeping the hydrophobic drug molecules in solution and delivering them to the surface of the biological membranes, where they partition into the membrane $(26,50)$. Though the complex does not penetrate the skin, the drug in the complex is in rapid dynamic equilibrium with the drug in the aqueous phase, thus continuously supplying the drug molecules to the skin surface in a diffusible form. On the other hand, CyDs act as penetration enhancers by increasing drug availability at the surface of the biological barrier and they are hypothesized to increase the drug transport through a kinetic barrier existing at the skin vehicle interface (50). In addition, drugs can be absorbed into the skin by aqueous pathway through hair follicles, sweat duct or intracellular aqueous pores in the stratum corneum (56-58); CyD complexation can increase drug flux through such aqueous pores or barriers (59).

Table 1 Effect of Enhancers on Permeation Rate of Glimepiride from the Investigated Chitosan Films

\begin{tabular}{|ll|}
\hline Flux $\left(\mathbf{m g} . \mathbf{c m}^{-2} \cdot \mathbf{h}^{-1}\right)$ & Enhancer \\
\hline $\mathbf{0 . 0 3 7} \pm \mathbf{0 . 0 0 4}$ & $-($ Glimepiride $)$ \\
\hline $\mathbf{0 . 0 6 7} \pm \mathbf{0 . 0 0 3} * * *$ & $-($ Glimepiride$-\beta-C y D)$ \\
\hline $\mathbf{0 . 0 3 8} \pm \mathbf{0 . 0 0 5}$ & $5 \%$ Oleic acid \\
\hline $\mathbf{0 . 0 6 1} \pm \mathbf{0 . 0 0 2} * * *$ & $5 \%$ Limonene \\
\hline
\end{tabular}




\begin{tabular}{|ll|}
\hline $\mathbf{0 . 0 6 2} \pm \mathbf{0 . 0 0 1} * * *$ & $10 \%$ Limonene \\
\hline $\mathbf{0 . 0 5 0} \pm \mathbf{0 . 0 0 2} * * *$ & $15 \%$ Limonene \\
\hline $\mathbf{0 . 0 3 7} \pm \mathbf{0 . 0 0 2}$ & $5 \%$ Cineole \\
\hline $\mathbf{0 . 0 3 1} \pm \mathbf{0 . 0 0 4} * *$ (Less) & $5 \%$ Menthol \\
\hline $\mathbf{0 . 0 6 3} \pm \mathbf{0 . 0 0 1} * * *$ & $5 \%$ Limonene $+10 \%$ Ethanol \\
\hline
\end{tabular}

Each value represents the mean \pm S.E. $(n=3)$

** Significant difference $\mathrm{p}<0.01$

$* * *$ Significant difference $\mathrm{p}<0.001$

Incorporation of $5 \%$ oleic acid in the chitosan film had no significant effect on the permeation rate of the drug $(p>0.05)$ (Table 1, Fig. 3).

Terpenes continue to be a popular choice as enhancers for delivering materials across skin membranes. It was recorded that mono- and sesquiterpenes are known to increase percutaneous resorption by increasing the diffusion in the stratum corneum and/or disruption of the intercellular lipid barrier (60, 61).

Incorporation of $5 \%$ limonene (Table 1, Fig. 3) led to significant enhancement $(p<0.001)$ of the flux of the drug from the film. The increment of limonene concentration to 10 and $15 \%$ also led to significant enhancement $(p<0.001)$ of the permeation rate of the drug from the film, however, these further increase in concentration did not induce further enhancement of drug flux as no significant difference $(p>0.05)$ was detected between the $5 \%$ and $10 \%$ concentrations of limonene. Even though, the enhancing effect of $15 \%$ limonene was significantly less than $5 \%$ limonene $(\mathrm{p}<0.001)$. This indicates that the permeation enhancement of limonene was concentration dependent and that the $5 \%$ concentration was the most potent.

Incorporation of 5\% cineole showed no significant enhancing effect $(\mathrm{p}>0.05)$. However, incorporation of $5 \%$ menthol even declined the permeation rate of glimepiride through rat skin (Table 1, Fig. 3).

It was reported that hydrocarbon terpenes such as limonene are generally the most effective terpene enhancers for lipophilic molecule such as steroids 54(Williams and Barry, 2004). Similar results were reported for the permeation of another lipophilic molecule, indomethacin, traversing rat skin (62); hydrocarbon terpenes, especially limonene, were as effective as Azone in promoting drug flux and oxygen containing terpenes (carvone, 1, 8cineole) were ineffective (63). Some structure activity relationships were apparent from that hydrocarbon terpenes were less potent enhancers for a model hydrophilic drug, 5-flourouracil, in human skin in vitro than were alcohol or ketone containing terpenes, and the greatest enhancement activity was shown by the oxide terpenes and terpenoids (60) However, such tentative structure activity relationships appear to be drug specific (54).

Ethanol is commonly used in many transdermal formulations and is often the solvent of choice for use in patches; as with water; ethanol permeates rapidly through human skin with a steady state flux of approximately $1 \mathrm{mg}$ $\mathrm{cm}-2 \mathrm{~h}^{-1}(64)$.

A combination of $5 \%$ limonene and $10 \%$ ethanol was tested to evaluate their mutual effect. It is evident that a further significant enhancement $(\mathrm{p}<0.05)$ was achieved compared to the volatile oil (Table 1, Fig. 3). This may be due to a synergistic enhancing effect due to facilitated incorporation of limonene into the stratum corneum.

Ethanol can exert its permeation enhancing activity through various mechanisms. Firstly, as a solvent, it can increase the solubility of the drug in the vehicle (65). Further, permeation of ethanol into the stratum corneum can alter the solubility properties of the tissue with a consequent improvement for drug partitioning into the membrane (66). Additionally, it is also feasible that the rapid permeation of ethanol or evaporative loss of this volatile solvent from the donor phase modifies the thermodynamic activity of the drug within the formulation.

On the basis of the above-presented results, chitosan films incorporating glimepiride in presence of $10 \%$ ethanol together with $5 \%$ limonene, as well as chitosan films incorporating glimepiride- $\beta$ - CyD complex exhibited distinctively marked high permeation rates.

Accordingly, three different transdermal formulae for glimepiride were selected for 
biological evaluation. These comprised: chitosan films containing glimepiride (formula 1), the same formula in presence of a combination of 5 $\%$ limonene together with $10 \%$ ethanol (formula 2) and chitosan film comprising glimepiride- $\beta$ CyD complex (formula 3). In addition, a plain chitosan film of the same size was also examined concurrently. The hypoglycemic effect of the selected formulations was assessed in diabetic rats and compared to oral administration of the drug.

\subsection{Assessment of Therapeutic Efficacy}

In vivo studies on diabetic rats revealed that BGL of the control group at different time intervals was higher than the corresponding level

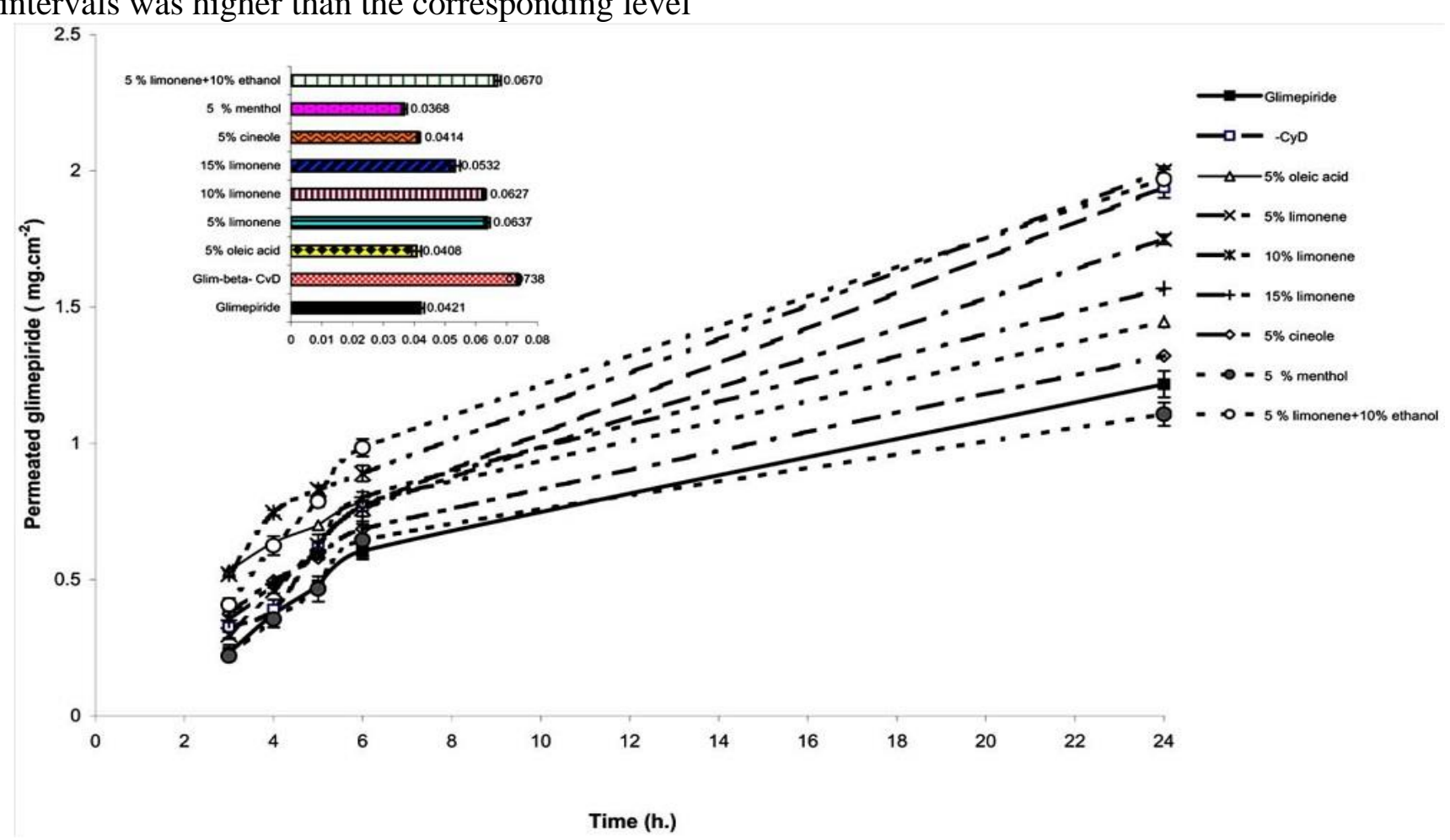

Figure 3. Effect of penetration enhancers on the permeation of glimepiride.
The peak of percentage decrease in BGL (Table 2) for the orally administered drug was higher than that for formulae 1; the difference is significant $(P<0.01)$. However, there is no significant difference $(P<0.05)$ between the oral drug and formulae 2 and 3 . This points out that the use of a combination of penetration enhancers, limonene and ethanol, or the use of glimepiride- $\beta$-CyD led to a significant increase in peak hypoglycaemic effect to such an extent reaching values comparable to that of the oral administration (Table 2 ).

The values of $t_{\max }$ for the transdermal formulae 1 and 2 were higher than that after oral for the groups treated by oral or transdermal administration of the drug (Fig. 4). All tested formulae exhibited significant $(\mathrm{p}<0.001)$ decrease in BGL compared to the plain chitosan film (control). The control group didn't show any noticeable hypoglycemic effect. Transdermal glimepiride formulae exhibited maximum hypoglycemic effect 4-6 hours post application and the hypoglycemic effect were sustained up to 48 hours. However, oral administration of the drug showed a peak percentage decrease in BGL, which was reached within 3-4 hours post dosing; after which a marked fast decline in hypoglycaemic effect was observed. administration of the drug the difference is significant $(P<0.01)$. On the other hand, there is no significant difference $(P<0.05)$ between the oral drug and formula 3 which contains glimepiride- $\beta$-CyD complex (Table 2).

The values of half peak time $\left(t_{1 / 2} p\right)$ for the investigated transdermal formulae presented in Table 2 were much higher than the corresponding values after oral administration, indicating prolongation of drug action; the difference is significant $(P<0.001)$. Regarding the area under the curve of percentage decrease in BGL versus time, the values of AUCO-48h for the transdermal formulae were higher than 
that of the orally administered drug; this difference is very highly significant, indicating that glimepiride-chitosan films afford adequate bioavailability of the drug (Table 2 ).

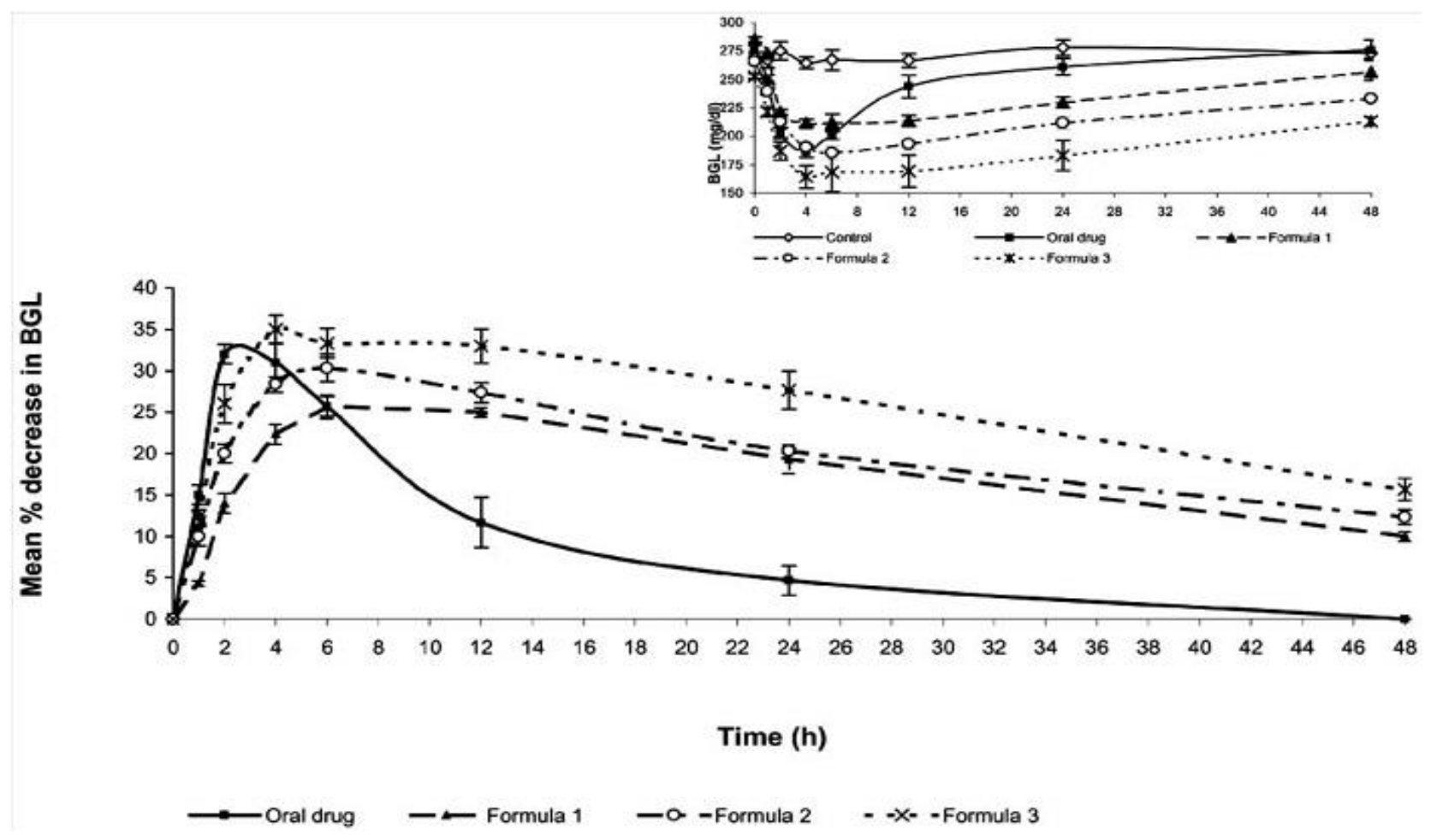

Figure 4. Percentage decrease in BGL after oral administration and transdermal application of glimepiride.

Table 2 Effect of Transdermal Formulations of Glimepiride on its Pharmacodynamic Parameters in Diabetic Rats

\begin{tabular}{|c|c|c|c|c|}
\hline \multirow[t]{2}{*}{ Treatment } & \multicolumn{4}{|c|}{ Pharmacodynamic Parameter } \\
\hline & $\begin{array}{c}\text { Max }(\%) \text { decrease in } \\
\text { BGL }\end{array}$ & $\mathbf{t}_{\max }(\mathbf{h r})$. & ${ }^{1} \mathbf{t}_{1 / 2 \mathbf{p}}(\mathbf{h r})$. & $\mathrm{AUC}_{(0-48 \mathrm{hr} .)}$ \\
\hline Oral glimepiride & $33.01 \pm 1.52$ & $3.33 \pm 0.70$ & $8.16 \pm 1.03$ & $399.42 \pm 29.06$ \\
\hline Formula 1 & $25.70 \pm 1.45 * *$ (Less) & $6.00 \pm 0.00 * *$ & $36.05 \pm 1.26 * * *$ & $860.42 \pm 47.42 * * *$ \\
\hline Formula 2 & $30.66 \pm 1.34$ & $5.33 \pm 0.67 * *$ & $36.83 \pm 1.31 * * *$ & $966.25 \pm 29.39 * * *$ \\
\hline Formula 3 & $35.20 \pm 1.73$ & $4.00 \pm 0.20$ & $37.12 \pm 1.258 * * *$ & $1228.64 \pm 86.09 * * *$ \\
\hline
\end{tabular}

$\mathrm{t}_{1 / 2 \mathrm{p}}$ : the time span during which the $\%$ decrease in blood glucose level of the drug is equal or higher than half its peak

** Significant difference $\mathrm{p}<0.01$

*** Significant difference $\mathrm{p}<0.001$

The above results demonstrate that chitosan films may offer an attractive, non-irritant, safe and economic transdermal delivery system for glimepiride. The prepared films are characterized by an intensity of action comparable to that of the orally administered drug accompanied with an increase in duration of action extending up to $48 \mathrm{~h}$.

\section{Conclusions}

In conclusion, transdermal delivery of glimepiride affords solution to many problems associated with oral administration problems. The annoyance of dizziness and gastric disturbances is avoided; consequently, patient compliance is increased. Transdermal delivery provides steadier basal blood drug levels due to prolonged delivery of the drug; thus, it prevents sub-therapeutic troughs and also reduces individual, variations. It avoids dangerous peaks which are the main cause of severe hypoglycaemia within the initial hours, in addition to the feasibility of termination of 
treatment when necessary. Transdermal glimepiride will help to maintain good glycaemic control for relatively prolonged period of time and, in turn, might help to prevent long term complications.

\section{Acknowledgement}

The authors are thankful to IJIST Journal for publishing their article.

\section{Conflicts of Interest}

The author declares that there are no conflicts of interest.

\section{References}

1. Kabadi, UM. Manag. Care, 2004, 13, 48.

2. Kouichi, I.; Masaki, W.; Youhei, N.; Takahiro, S.; Nobuki, T; Masahiko, T.; Hideyuki, K.; Kensuke, Y.; Masao, S., Susumu, K.;Takuya A.; Shigehiro, K. Diabetes Res. Clin. Pract., 2005, 68, 250.

3. Thummel, K.E. Adv. Drug Deliv. Rev., 1997, $27,97$.

4. Frick, A.; Moller H.; Wirbitzki, E. Eur. J. Pharm. Biopharm., 1998, 46, 305.

5. Massimo, M.B. Clin. Ther., 2003, 25, 799.

6. Profozic V.L.; Metelko, Z.L. Dis. Manag. Health Outcomes, 1998, 4, 29.

7. Barry, B. W. Eur. J .Pharm. Sci., 2001, 14, 101.

8. Panchagnula, R., Ind. J. Pharm., 1997, 29, 140.

9. Takahashi, Y.; Furuya, K.; Iwata, M.; Onishi, H.; Machida, Y.; Shirotake, S. Yakugaku Zasshi, 1997, $117,1022$.

10. Takahashi, Y.; Iwata, M.; Machida, Y. Yakugaku Zasshi, 2001. 121, 161.

11. Mutalik, S.; Udupa, N. J. Pharm. Sci., 2004, 93, 1577.

12. Sridevi, S.; Diwan, P.V.R. Pharmazie, 2002, 57, 632.

13. Mutalik, S.; Udupa, N. Pharmazie, 2002, 57, 838.

14. Ammar, H.O; Salama, H.A.; Ghorab, M.; El-Nahhas S.; Elmotasem, H. Curr. Drug Deliv., 2006, 3, 333.

15. Zhang, Y.; Xu, D.H.; Ma, Z.; Chen, Y.; Zhao, J.J; Xu, S.B. Yao Xue Xue Bao., 2004, 39, 640.

16. Chae, Su.Y.; Jang, Mi.K.; Nah, J.W. J. Control. Release, 2005, 102, 383.

17. Paul, W.; Sharma, C.P. S.T.P. Pharm. Sci., 2000,10, 5.

18. Illum, L., Pharm. Res., 1998, 15, 1326.

19. Ranaldi, G.; Marigliano, I.; Vespignani, I.; Perozzi, G.; Sambuy, Y. J. Nutr. Biochem., 2002, 13, 157.

20. Schipper, N.G.M.; Olsson, S.; Hoogstraate, J.A.; deBoer, A.G.; Varum, K.M.; Artursson, P. Pharm. Res., 1997, 14, 923.

21. Schipper, N.G.M.; Varum, K.M.; Stenberg, P.; Ocklind, G.; Lennernas, H.; Artursson, P. Eur. J. Pharm. Sci., 1999, 8, 335.

22. Zerrouka, N; Menninib, N.; Maestrellib, F.; Chemtoba, C.; Murab, P. Eur. J. Pharm. Biopharm., 2004, 57, 93.

23. Foldvari, M. Pharm.Sci. Technol. Today., 2000, 3, 417.

24. Ammar, H.O.; Salama, H.A.; Ghorab M.; Mahmoud, A.A. Int. J. Pharm., 2006, 309, 129.
25. Ammar, H.O.; El-Nahhas, S.A. Pharmazie, 1995, 50, 408.

26. Loftsson, T.; Bodor, N. Effect of cyclodextrins on percutaneous transport of drugs. In: Smith, E.W.; Maibach, H.I., Eds, Percutaneous Penetration Enhancers. Florida. CRC Press, Boca Raton, 1995, pp. 335.

27. Mastuda, H.; Arima, H. Adv. Drug Deliv. Rev., 1999, 36, 81.

28. Lopez, R.F.; Colett, J.H.; Bently, M.V. Int. J. Pharm., 2000, 200, 27.

29. Martin, A.; Bustamante, P.; Chun A.H.C. Physical Pharmacy: Physical Chemical Principles in Pharmaceutical Sciences (forth ed.), Lippincott Williams \& Wilkins, 1993, pp. 260.

30. Uekema, K.; Horiuchi Y.; Kikuchi M. J. Incl. Phenom., 1988, 6, 167.

31. El-Sabbagh, S.H.; Hussain A.I.; Abd El-Ghaffar, M.A. Pigment \& Resin Technol., 2005, 4, 203.

32. Shah, V.P.; Tymes, N.W.; Yamamoto, L.A.; Skelly, J.P. Int. J. Pharm., 1986, 32, 243.

33. Vogel, H.G.; Vogel, W.H. Drug Discovery and Evaluation: Pharmacological Assays. Berlin. Springer - Verlag, 1997, pp. 570.

34. Stepensky, D.; Friedman, M.; Srour, W.; Raz, I.; Hoffman, A., J. Control. Release, 2001, 71, 107.

35. Pepato, M.T.; Keller, E.H; Baviera, A.M.; Kettelhut, I.C.; Vendarmini, R.C. J. Ethnopharmacol., 2002, 81, 191.

36. Gabra, P.H.; Sirois, P. Eur. J. Pharm., 2005, 514, 61.

37. Ladriere, L.; Malaisse-Lagae, F.; Fuhlendorff, J.; Malaisse, W.J. Eur. J. Pharm., 1997, 335, 227.

38. Wagner, S.G., Fundamentals of Clinical Pharmacokinetics (first ed.), Drug Intelligence Publications Inc., Hamilton, Illinois, 1975, pp. 7.

39. Knapczyk, J. Int. J. Pharm., 1993, 93, 233.

40. Lai, H.M.; Padua, G.W. Cereal. Chem., 1997, 74, 771.

41. Wu, C.; McGinity, J.W. Int. J. Pharm., 1999, 177, 15.

42. Peppas, N.A.; Buri, P.A. J. Control. Release, 1985, 2, 257.

43. Ahmad Khan, T.; Peh, K. K.; Ching, H. S. Pharm. Pharmaceut. Sci., 2000, 3, 303.

44. Kurozumi, M.; Nambu, N.; Nagai, T. Chem. Pharm. Bull., 1975, 23, 3062.

45. Ozkana, Y.; Ataya, T.; Dikmena, N.; I $\square$ imera, A.; Aboul-Enein, H.Y. Pharm. Acta Helv., 2000, 74, 365.

46. Fernandes, M.; Vieira M.T.; Veigaa, F. Eur. J. Pharm. Sci., 2002, 15, 79

47. Jug, M.; Becirevic-Lacan, M. Eur. J. Pharm. Sci., 2004, 21, 251.

48. Ryan, J.A. J. Pharm. Sci., 1986, 75, 805.

49. McCrum, N.G., Buckley, C.P., Bucknall, C.B. (Eds).Principles of Polymer Engineering. Oxford University Press, New York, 1988, pp. 18.

50. Loftsson, T.; Massom, M. Int. J. Pharm. 2001, 225, 15 .

51. Larrucea, E.; Arellan, A.; Santoyo, S.; Ygartu, P. Drug Dev. Ind. Pharm., 2002, 28, 245.

52. Sridevi, S.; Diwan, P.V.R. Eur. J. Pharm. Biopharm., 2002, 54, 151.

53. Uekama, K.; Hirayama, F.; Irie T. Chem. Rev., 1998, 98, 2045. 
54. Williams, A.C.; Barry, B.W. Adv. Drug Deliv. Rev., 2004, 56, 603 .

55. Loftsson, T.; Olafsson, J.H. Int. J. Dermatol., 1998, 37, 241.

56. Meidan, M.V.; Docker, M.; Walmsley A.D.; Irwin, W.J. Pharm. Res., 1998, 15, 85.

57. Illel, H.; Schaefer, J.; Wepierre, J.; Doucet, O. J. Pharm. Sci., 1991, 80, 424.

58. Li, S.K.; Suh, W.; Parikh, H.H.; Ghanem, A.H.; Mehta, S.C.; Peck, K.D.; Higuchi W.I. Int. J. Pharm., 1998, 170, 93.

59. Preiss, A.; Mehnert, W.; Fromming, KH. Pharmazie, 1995, 50, 121.

60. Williams, A.C.; Barry, B.W. Int. J. Pharm., 1991, 74, 157.

61. Cornwell, P.A.; Barry, B.W. Int. J. Pharm., 1993, 94, 189.

62. Okabe, H.; Takayama, K.; Ogura, A.;Nagai T. Drug Des. Deliv., 1989, 4, 313.

63. Nagai, T.; Okabe, H.; Ogura, A.; Takayama, K. Effect of limonene and related compounds on the percutaneous absorption of indomethacin. In: Proceedings of the 16th International Symposium on Controlled Release Bioactive Material, Chicago, USA. 1989, pp. 181.

64. Berner, B.; Mazzenga, G.C; Otte, J.H.; Steffens. R.J.; Juang R.H.; Ebert, C.D. J. Pharm. Sci., 1989, 78, 402.

65. Pershing, L.K.; Lambert, L.D.; Knutson, K. Pharm. Res., 1990, 7, 170.

66. Megrab, N.A.; Williams A.C.; Barry, B.W. Int. J. Pharm., 1995, 116, 101. 